\section{Severe congestive heart failure in elderly patient with Paget's disease}

\author{
Lorenzo Palleschi, ${ }^{1}$ Eleonora Nunziata ${ }^{2}$ \\ ${ }^{1}$ Unit of Geriatrics; and ${ }^{2}$ Department of \\ Emergency, A.O. S. Giovanni-Addolorata, \\ Rome, Italy
}

\begin{abstract}
Paget's disease of bone is characterized by focal areas of increased bone turnover, affecting one or several bones throughout the skeleton. Paget's disease is often asymptomatic, but can be associated with bone pain and other skeletal and non-skeletal complications. We report a case of a 84-year old woman who presented a clinical condition suggesting Paget's disease of bone complicated by congestive heart failure.
\end{abstract}

\section{Introduction}

Paget's disease of bone (osteitis deformans) is a metabolic bone disease that usually begins after the fourth decade and affects bone remodeling and is characterized by the excessive bone resorption followed by abnormal bone formation, affecting one or several bones throughout the skeleton. ${ }^{1,2}$ Excessive osteoclastic bone resorption followed by a secondary increase in osteoblastic activity leads to replacement of the normal bone by a disorganized, enlarged, and weakened osseous structure that is prone to deformities and fractures.

Paget's disease (PD) probably occurs equally often in men and women and clearly increases in prevalence with age. Genetic factors have an important role in this disease, and environmental factors are involved too. ${ }^{1,2}$

Bisphosphonates, structural stable analogs of pyrophosphate, are potent inhibitors of bone resorption and have been used successfully for the treatment of Paget's disease. ${ }^{3,4}$

We describe a case of PD of bone in a 84-year old woman.

\section{Case Report}

An 84-year old woman was admitted to geriatric unit from the emergency room for severe pain in multiple vertebral fractures, left ilio and ischio-pubic branches fracture and sacral fracture. She had history of arterial hypertension, severe osteoporosis, cognitive impairment with behavioral and psychological symptoms of dementia (BPSD).

At the time of admission she was taking verapamil $80 \mathrm{mg} / \mathrm{die}$, lisinopril $20 \mathrm{mg} / \mathrm{die}$, alendronate $70 \mathrm{mg} /$ week, vitamin D 25,000 UI/week.

Routine laboratory analyses were normal except for a slight reduction in hemoglobin $(10.8 \mathrm{~g} / \mathrm{dL})$, in serum calcium (7.9 $\mathrm{mg} / \mathrm{dL})$ and in albumin $(2.65 \mathrm{~g} / \mathrm{dL})$ levels. Serum alkaline phosphatase (ALP) level was $67.56 \mathrm{IU} / \mathrm{L}$ (reference range, 3-15 $\mu \mathrm{g} / \mathrm{L}$ ). Tumor markers (CA 125, CA 15.3, CA 19.9, CEA, NSE) and parathyroid hormone (PTH) were normal. Total body scan, made in the suspicion of metastatic cancer, confirmed skeletal metastatic disease with pathological fractures of ribs, sternum, lumbar vertebrae (L1-L2), left ilio and ischiopubic branches (Figure 1).

During hospitalization the patient became dyspneic and the electrocardiogram revealed atrial fibrillation which was treated with amiodarone, but the patient got worse and was admitted to cardiac care unit for acute pulmonary edema. The electrocardiogram showed sinus rhythm and left bundle branch block. Troponin increased. Echocardiography revealed left ventricular hypertrophy and reduced left ventricular function (E.F. 30\%). The patient was treated with non-invasive ventilation, diuretics, nitrate.

After four days the patient improved and was transferred back to geriatrics care unit, where the bone biopsy was performed, because of the suspicion of metastatic cancer.

The histological examinations showed a bone remodeling with localized excess of osteoclastic bone resorption and osteoblasts on surfaces of new bone formation. There were not invasive tumor cells.

Diagnosed with Paget bone disease the patient was discharged with oral residronate $35 \mathrm{mg}$ once daily for 3 months and vitamin D 25,000 UI once a week. When patient came back home, she had a severe limitation of physical activity and was dyspneic at rest (NHYA IV). Mini-mental state examination (MMSE) revealed a moderately severe cognitive impairment (MMSE: 19/30), furthermore the assessment of daily routine activity showed a functional disability (ADL $1 / 6$, IADL 0/8).

The follow-up visit, three months after oral treatment of residronate, showed improving patient's clinical conditions, disappearing of severe pain, blood pressure at $120 / 60 \mathrm{mmHg}$ and an electrocardiogram indicating sinus rhythm heart rate at $62 \mathrm{bpm}$. The patient had a slight limitation of the ordi-
Correspondence: Eleonora Nunziata,

Department of Emergency, A.O. S. GiovanniAddolorata, Rome, Italy.

E-mail: ele.nunziata@gmail.com

Key words: Paget's disease; diphosphonates; alkaline phosphatase.

Contributions: LP made substantial contributions to conception and design, and acquisition of data. He gave final approval of the version to be submitted; EN participated in drafting the article, revising it critically for important intellectual content.

Received for publication: 27 March 2017 Accepted for publication: 20 April 2017.

This work is licensed under a Creative Commons Attribution-NonCommercial 4.0 International License (CC BY-NC 4.0).

CC Copyright L. Palleschi and E. Nunziata, 2017 Licensee PAGEPress, Italy

Geriatric Care 2017; 3:6727

doi:10.4081/gc.2017.6727

nary physical activity (NYHA II) due to the onset of dyspnea during the course of the said activity. Serum alkaline phosphatase (AP) decreased (15.7 IU/L). The echocardiography showed a left atrial enlargement without ventricular hypertrophy and normal left ventricular function (E.F. 55\%).

\section{Discussion}

In Western Countries, Paget's bone disease is one of the priorities in differential diagnosis for elderly patients who present osteoblastic lesions. Most patients had polyostotic disease $(72 \%)$, and the pelvis (67\%), vertebra (41\%), and femur (31\%) were the most common sites of involvement. Skeletal complications attributable to Paget's disease included bowing deformities $(7.6 \%)$, fracture of pagetic bone $(9.7 \%)$, and osteosarcoma $(0.4 \%)$. Osteoarthritis was observed in $73 \%$ of patients, and $11 \%$ had a hip or knee replacement. Non-skeletal complications related to Paget's disease included cranial nerve $(0.4 \%)$, peripheral nerve $(1.7 \%)$, and nerve root $(3.8 \%)$ compression, basilar invagination (2.1\%), hypercalcemia $(5.2 \%)$, and congestive heart failure $(3.0 \%)$ and hearing loss $(61 \%) .5,6$

The diagnosis of Paget's disease require a high index of suspicion and conditions which could cause sclerosis of the bone like metastasis from solid tumors like breast, prostate and lung need to be excluded. ${ }^{7}$

Three tests (an X-ray, a blood test, and 
a bone scan) are commonly used to discover Paget's disease. X-rays are used to determine the final diagnosis. In X-ray images, bones affected by Paget's disease have a particular appearance. Radiographs include both lytic (early) and sclerotic findings,
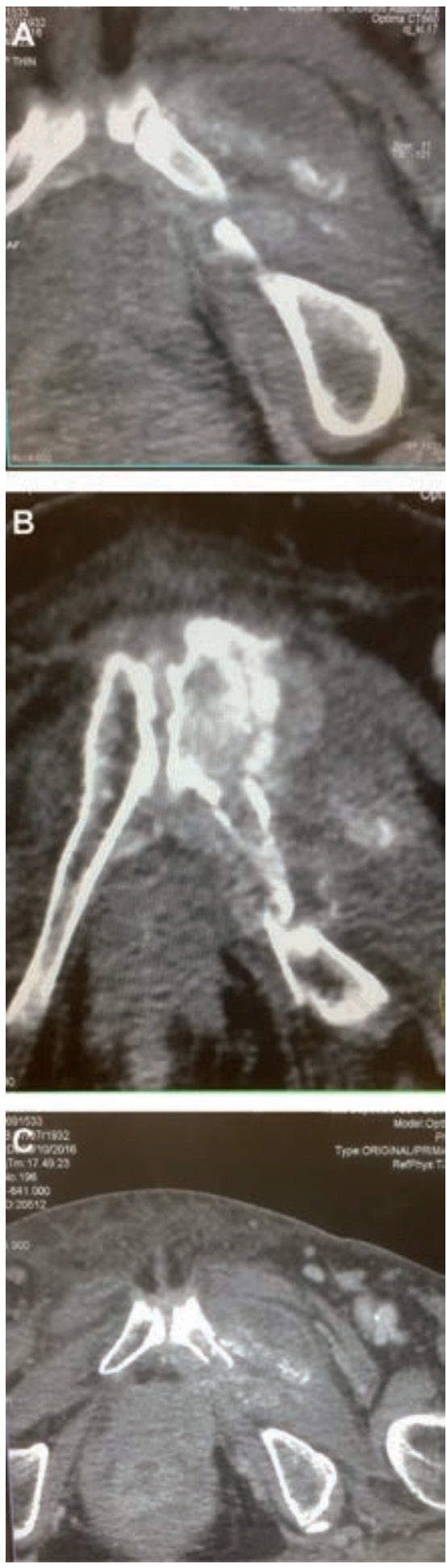

Figure 1. Pathological fractures of pelvis. osteoporosis circumscripta in skull, bowed limbs, and flame-shaped lesions in long bones. Many patients are diagnosed incidentally in the asymptomatic phase through plain radiographs that show localized enlargement of bone. These radiographs often have a high specificity because of their classic nature, but a low sensitivity. In a blood test, a higher level of serum AP is a sign that the disease may be present. An AP level greater than twice the usual level strongly suggests Paget's disease, especially if the patient's serum calcium level, phosphorus level, PTH level and kidney function are normal. Increased bone turnover and remodeling is associated with elevated levels of serum AP. Such a marker allows an earlier diagnostic and assessment of the activity of Paget's disease and monitoring of the effectiveness of the medical treatment by bisphosphonates. ${ }^{8,9}$

A bone scan helps identify which bones have been affected by Paget's disease. Furthermore, bone scans can be used to increase diagnostic sensitivity in patients suspected of having Paget's disease, although this method is less specific and should be interpreted cautiously.

A hallmark of the pathology of Paget's disease is the increased vascularity of affected bones. Further evidence for this has been documented by demonstration of an increase in blood flow to the extremities, ${ }^{10}$ although it has been suggested that this is mainly caused by cutaneous vasodilation. ${ }^{11}$ An echocardiographic study of cardiac function in Paget's disease found that patients with more severe disease had lower peripheral vascular resistance and higher stroke volume. ${ }^{12}$ These observations help account for the finding that patients with $15 \%$ or more of their skeleton affected by Paget's disease have increased cardiac output. ${ }^{13}$

High-output heart failure (HF) is characterized by an elevated resting cardiac index beyond the normal range of 2.5 to 4 $\mathrm{L} / \mathrm{min}$ per $\mathrm{m}^{2}$. Ineffective blood volume and pressure, chronic activation of the sympathetic nervous system and reninangiotensin-aldosterone axis, increased serum vasopressin (antidiuretic hormone) concentrations, and chronic volume overload gradually cause ventricular enlargement, remodeling, and HF. ${ }^{14}$ A number of conditions lead to an obligatory increase in cardiac output, which can be associated with HF in some patients.

In most patients with high-output HF, high cardiac output provokes HF in the setting of reduced ventricular reserve from some underlying cardiac problem. ${ }^{14,15}$

Concerning our patient case study we assumed that congestive HF with a low cardiac output was occasioned by an underly- ing coronary artery disease and atrial fibrillation in circumstance of high-output heart failure. Unfortunately we did not perform an echocardiography before the hospitalization, consequently we could not observe the hemodynamic parameters before the occurrence of acute pulmonary edema.

Furthermore, following the oral treatment of risedronate, patients' health status and physical performance improved, as a matter of fact the new echocardiography showed a significant regression of heart failure symptoms. Finally, our patient did not experienced any side effects after three months of therapy.

Medical management of Paget's disease of bone is based on giving inhibitors of osteoclastic bone resorption, and bisphosphonates are the treatment of first choice ${ }^{16}$. Bisphosphonate therapy is primarily indicated for patients who have bone pain arising from increased metabolic activity in affected bones. ${ }^{16}$ Bisphosphonate therapy is highly effective at reducing bone turnover, and it has been shown to heal radiological lesions and restore normal histology. ${ }^{17,18}$

\section{Conclusions}

In conclusion this case demonstrated an older patient with Paget's disease, complicated by congestive heart failure, who had been effectively treated with oral bisphosphonates.

\section{References}

1. Papapoulos SE. Paget's disease of bone: clinical, pathogenetic and therapeutic aspects. Ballieres Clin Endocrinol Metab 1997;11:117-43.

2. Ooi CG, Fraser WD. Paget's disease of bone. Postgrad Med J 1997;73:69-74.

3. Delmas PD, Meunier PJ. The management of Paget's disease of bone. N Engl J Med 1997; 336:559-66.

4. Siris ES. Extensive personal experience: Paget's disease of bone. J Clin Endocrinol Metab 1994; 80:335-8.

5. Cundy T, Reid IR. Paget's disease of bone. Clin Biochem 2012;45:43-8.

6. Wick MR, Siegal GP, Unni KK, et al. 3rd Sarcomas of bone complicating osteitis deformans (Paget's disease): fifty years' experience. Am J Surg Pathol 1981;5:47-59.

7. Kanis JA. Pathophysiology and treatment of Paget's disease of bone. 2nd ed. London: Martin Dunitz; 1998.

8. Reid IR, Davidson JS, Wattie D, et al. 
Comparative responses of bone turnover markers to biphosphonates therapy in Paget's disease of bone. Bone 2004;35:224-30.

9. Alvarez L, Peris P, Pons F, et al. Relationship between biochemical markers of bone turnover and bone scintigraphic indices in assessment of Paget's disease actuvity. Arthritis Rheumatol 1997;40:461-8.

10. Edholm O, Howarth S. Studies on the peripheral circulation in osteitis deformans. Clin Sci 1953;12:277-85.

11. Heistad DD, Abboud FM, Schmid PG, et al. Regulation of blood flow in
Paget's disease of bone. J Clin Invest 1975;55:69-74.

12. Morales-Piga AA, Moya JL, Bachiller FJ, et al. Assessment of cardiac function by echocardiography in Paget's disease of bone. Clin Exp Rheumatol 2000; 18:31-7.

13. Arnalich F, Plaza I, Sobrino JA, et al. Cardiac size and function in Paget's disease of bone. Int J Cardiol 1984;5:491505 .

14. Wasse H, Singapuri MS. High-output heart failure: how to define it, when to treat it, and how to treat it. Semin Nephrol 2012;32:551.
15. Sy AO, Plantholt S. Congestive heart failure secondary to an arteriovenous fistula from cardiac catheterization and angioplasty. Cathet Cardiovasc Diagn 1991;23:136.

16. Lyles KW, Siris ES, Singer FR, et al. A clinical approach to diagnosis and management of Paget's disease of bone. J Bone Miner Res 2001;16:1379-87.

17. Cundy T, Reid IR. Paget's disease of bone. Clin Biochem 2012;45:43-8.

18. Ralston SH, Langston AL, Reid IR. Pathogenesis and management of Paget's disease of bone. Lancet 2008; 372:155-63 